\title{
Evaluation of Peripapillary Retinal Nerve Fiber Layer by Optical Coherence Tomography in Children with Iron Deficiency Anemia
}

\author{
Abdelmonem M. Hamed, Mohamed R. El Sayed, Mohamed A. Heikal, Ehsan Abd Al Basset
}

Department of Ophthalmology,

Benha faculty of medicine,

Banha University, Egypt.

Correspondence to: Ehsan Abd Al Basset, Department of Ophthalmology Benha faculty of medicine, Banha University, Egypt.

\section{Email:}

ehsanabasset@gmail.com

Received: 20 October 2019

Accepted: 25 December 2021

\begin{abstract}
Purpose. To evaluate peripapillary retinal nerve fiber layer (RNFL) thickness in children with iron deficiency anemia (IDA) in comparison with healthy controls and to investigate the correlation between peripapillary RNFL thicknesses and the hematologic parameters in these subjects. Methods. 16 children with a diagnosis of IDA (anemic group) and 16 children of age- and sex-matched healthy children (control group) were enrolled in this study. The measurement of peripapillary RNFL thickness was obtained by Topcon 3D spectral domain OCT 2000 Fa. Results. Mean age of control group and anemic one was $10 \pm 3$ and $10 \pm 2$ years respectively. average RNFL and RNFL of inferior, superior, nasal and temporal quadrants were thinner in children with IDA than in healthy children ( $\mathrm{P}$ value $<0.001,<0.001$, 0.001, 0.002 and 0.011 respectively), In addition, average peripapillary RNFL thickness and RNFL thicknesses of inferior, superior, and temporal quadrants were correlated with hemoglobin levels in anemic children $\left(\mathrm{r}_{1}=0.500, \mathrm{P}_{1}<0.049, \mathrm{r}_{2}=0.560, \mathrm{P}_{2}<0.024, \mathrm{r}_{3}=0.506, \mathrm{P}_{3}\right.$ $<0.046, \mathrm{r}_{4}=0.654, \mathrm{P}_{4}=0.006$, respectively). Conclusions. This study showed that children with IDA had different peripapillary RNFL
\end{abstract} profile measured by Topcon 3D spectral domain OCT-2000 Fa. We caution ophthalmologists when they measure RNFL thickness in children to diagnose glaucoma or other neuro-ophthalmic disorders.

Key words: Retinal Nerve Fiber Layer, Children 


\section{Introduction}

Iron is essential for the normal development and function of all tissues in the body. Ironcontaining heme proteins (hemoglobin and cytochromes) participate in tissue oxygen delivery and energy metabolism. In the brain, iron and iron-containing enzymes are necessary for neuronal and glial energy metabolism, myelin synthesis and neurotransmission. Also, the iron is necessary for the structural stability and maintenance of the optic nerve and for photo transduction in the retina. From a public health point of view, iron deficiency is the most common micronutrient deficiency in the world and the number of people, who suffer it, is estimated to reach over one billion and includes individuals of lower economic class. Preschool age children and women of childbearing age and are particularly vulnerable. In addition to being the most common cause of anemia, iron deficiency during the late prenatal and early postnatal periods is a risk factor for longterm neurodevelopmental abnormalities (1)(2).

Iron deficiency has also been found to be associated with dopaminergic dysfunction. Dopamine is a well-known major neurotransmitter or modulator in the retina (3), which its deficiency is believed to cause modification of receptive field properties of ganglion cells which form retinal nerve fiber layer (RNFL) by their axons (4).

The occurrence of retinopathy in patients suffering from severe anemia is well known. The most frequent symptoms are retinal hemorrhages and soft exudates. Ischemic retinopathy, venous tortuosity and papilledema have also been described, and the exact mechanism leading to fundus lesions is still not completely understood, but it seems to be related to retinal hypoxia (5).

A pervious study showed that infants with IDA had reduced spontaneous eye-blinking rate, which has been found to be related to dopaminergic neurotransmission (3).

Optical coherence tomography (OCT) is a noninvasive imaging method that is widely used in the diagnosis of optic nerve and RNFL diseases. It has enabled in vivo quantitative analysis of peripapillary RNFL. The spectral domain OCT technique provides faster acquisition, better resolution and improved imaging of retinal morphology than traditional OCT (6). 
Several studies have demonstrated that IDA was associated with thinning of peripapillary RNFL (4) (6) (7) (8) and to our knowledge there is no studies, in this context, was conducted in Egyptian children that suffer from IDA.

And so, in this study we aimed to evaluate peripapillary RNFL thickness in children with IDA of Egyptian population and to be compared with healthy controls and to find whether the peripapillary RNFL thicknesses is correlated with other hematologic parameters or not.

\section{Materials and methods}

This is a case control study and was conducted in the period from November 2017 to November 2018, at Ophthalmology Department, Benha University Hospital. 16 children with a diagnosis of IDA (anemic group) and 16 eyes of age- and sex matched healthy children (control group) were consecutively enrolled in this study. The IDA was diagnosed as hemoglobin <11.5 $\mathrm{g} / \mathrm{dL}$, mean corpuscular volume (MCV) $<77$ $\mathrm{fl}$, serum ferritin $<20 \mathrm{ng} / \mathrm{mL}$, serum iron $<20$ $\mathrm{ug} / \mathrm{dL}$ and serum Total iron binding capacity (TIBC) over $450 \mathrm{ug} / \mathrm{dL}$. All subjects and parents were informed about the study and the parents of all participants consented to participate. Each subject underwent a standard ophthalmologic examination including determination of refractive error and best corrected visual acuity (BCVA), slit-lamp examination, measurement of intra ocular pressure (IOP) by Topcon CT-80 computerized tonometer (Topcon Medical Systems Inc, Tokyo, Japan), funduscopy and pupillary light reflex. A detailed clinical examination was also performed for each subject.

Exclusion criteria included, history of any previous refractive, ocular surgery and ocular injuries, corneal opacities, corneal dystrophies and any corneal diseases, retinal diseases including retinal degenerations and dystrophies, an intraocular pressure higher than $21 \mathrm{mmHg}$ in one or both eyes, myopia or hyperopia more than 6.0 diopters, strabismus, glaucoma, neurological diseases and uncooperative children for RNFL imaging.

Peripapillary RNFL thickness measurements were performed using Topcon 3D OCT-2000 Fa (Topcon Medical Systems Inc, Tokyo, Japan), with pupil dilation of the subjects. Peripapillary RNFL thicknesses of temporal, nasal, inferior, and superior quadrants and average RNFL thickness were obtained with optic disc 6x6mm 3D disc scan Protocol. 


\section{Stastical analysis}

Data management and statistical analysis were done using SPSS vs.25. (IBM, Armonk, New York, United states).

Numerical data was summarized as means and standard deviations or medians and ranges. Categorical data was summarized as numbers and percentages.

Comparisons between two groups were done using Mann Whitney $U$ test for numerical data and Chi-square test for categorical data. Nonparametric tests for numerical data were planned due to relatively small numbers in both groups.

Correlation analysis was done between RNFL thicknesses and hematological parameters using Spearman's correlation.
" $\mathrm{r}$ " is the correlation coefficient. It ranges from -1 to +1 . -1 indicates strong negative correlation. +1 indicates strong positive correlation and 0 indicates no correlation. All $\mathrm{P}$ values were two sided. $\mathrm{P}$ values less than 0.05 were considered significant.

\section{Results}

The mean age of control group and anemic one was $10 \pm 3$ and $10 \pm 2$ years respectively. As presented in table (1), there were no significant differences between both groups as regard IOP, BCVA and SE. P values were $0.361, \quad 0.381$ and 0.642 respectively. whereas they were significantly different regarding hemoglobin, serum iron, ferritin, $\mathrm{MCV}$, and total iron binding capacity (all $\mathrm{p}<0.001)$ table (2).

Table (1): IOP, BCVA and SE in both groups

\begin{tabular}{|c|c|c|c|c|}
\hline & & Cases $(n=16)$ & Controls $(n=16)$ & $P$ value \\
\hline IOP (mmHg) & Mean \pm SD & $15.9 \pm 2.1$ & $16.7 \pm 1.8$ & 0.361 \\
\hline BCVA & Median (range) & $0.95 \pm 0.09$ & $0.93 \pm 0.09$ & 0.381 \\
\hline SE & Median (range) & $0.59(-3.88-2.06)$ & $0.22(-2.06-1.44)$ & 0.642 \\
\hline
\end{tabular}

Mann Whitney U test was used. SD = Standard deviation, IOP = Intra ocular pressure, BCVA = Best corrected visual acuity, $\mathrm{SE}=$ Spherical equivalent 
Table (2) Hematological parameters in both groups

\begin{tabular}{lccccc}
\hline & \multicolumn{2}{c}{ Cases $(\mathbf{n}=\mathbf{1 6})$} & \multicolumn{2}{c}{ Controls $(\mathbf{n}=\mathbf{1 6})$} & $\mathbf{\pm}$ \\
& Mean & $\mathbf{\pm S D}$ & Mean & $\mathbf{\pm S D}$ & P value \\
\hline Hemoglobin (g/dl) & 10 & 0.6 & 12.4 & 0.4 & $<0.001$ \\
Iron (ug/dl) & 18 & 5 & 90 & 6 & $<0.001$ \\
Ferritin (ng/ml) & 14.7 & 3.43 & 201.99 & 26.38 & $<0.001$ \\
TIBC (ug/dl) & 456 & 6 & 273 & 16 & $<0.001$ \\
MCV (fl) & 67.7 & 4.5 & 85 & 3.8 & $<0.001$ \\
\hline
\end{tabular}

Mann Whitney U test was used. SD = Standard deviation, TIBC = Total iron binding capacity, MCV = Mean corpuscular volume

The total RNFL and RNFL of inferior, superior, nasal and temporal quadrants were thinner in children with IDA than in healthy children $(\mathrm{P}$ value $<0.001,<0.001,0.001,0.002$ and 0.011 respectively) table (3) and figure (1).

Table (3) Peri-papillary retinal nerve fiber layer thickness in both groups

\begin{tabular}{lccccc}
\hline & \multicolumn{2}{c}{ Cases $(\mathbf{n}=\mathbf{1 6})$} & \multicolumn{2}{c}{ Controls $(\mathbf{n}=\mathbf{1 6})$} & \\
& Mean & $\pm \mathbf{S D}$ & Mean & $\mathbf{\pm S D}$ & P value \\
\hline Total thickness (um) & 92.2 & 7.2 & 107.8 & 5.4 & $<0.001$ \\
Inferior quadrant (um) & 110.9 & 17.2 & 137.4 & 8.5 & $<0.001$ \\
Superior quadrant (um) & 112.4 & 14.5 & 127.8 & 11.3 & 0.001 \\
Nasal quadrant (um) & 75.4 & 13 & 86.7 & 10 & 0.002 \\
Temporal quadrant & 70.7 & 8.9 & 80.2 & 11.4 & 0.011 \\
\hline
\end{tabular}

Mann Whitney U test was used. SD = Standard deviation

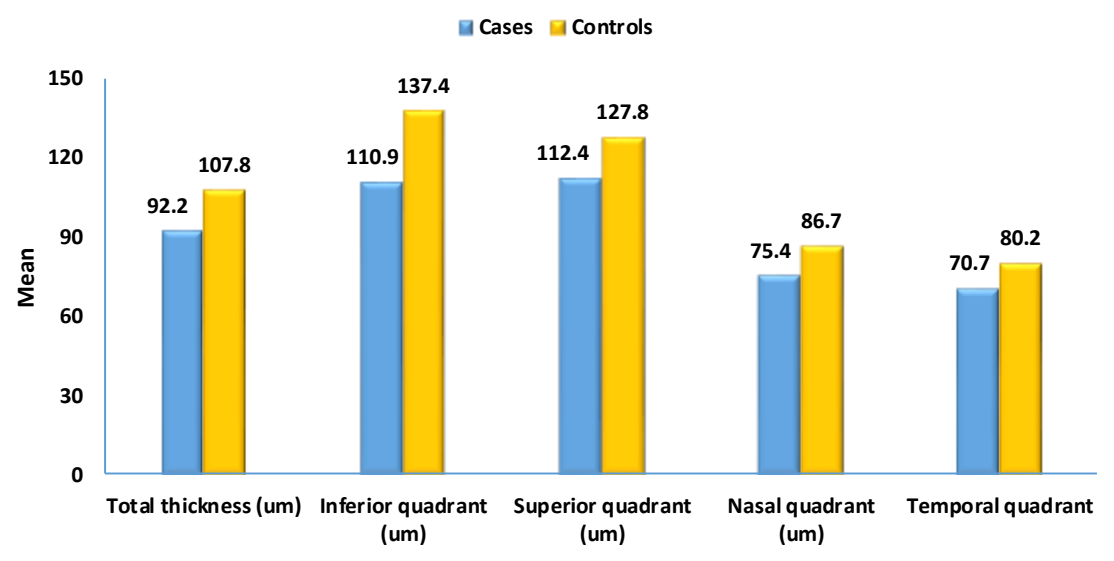

Figure (1) Peri-papillary retinal nerve fiber layer thickness in both groups 
The total peripapillary RNFL thickness and RNFL thicknesses of inferior, superior, and temporal quadrants were correlated with hemoglobin levels in anemic children $\left(\mathrm{r}_{1}=0.500, \mathrm{P}_{1}\right.$ $<0.049, \mathrm{r}_{2}=0.560, \mathrm{P}_{2}<0.024, \mathrm{r}_{3}=0.506, \mathrm{P}_{3}<0.046, \mathrm{r}_{4}=0.654, \mathrm{P}_{4}=0.006$, respectively) figures (2).

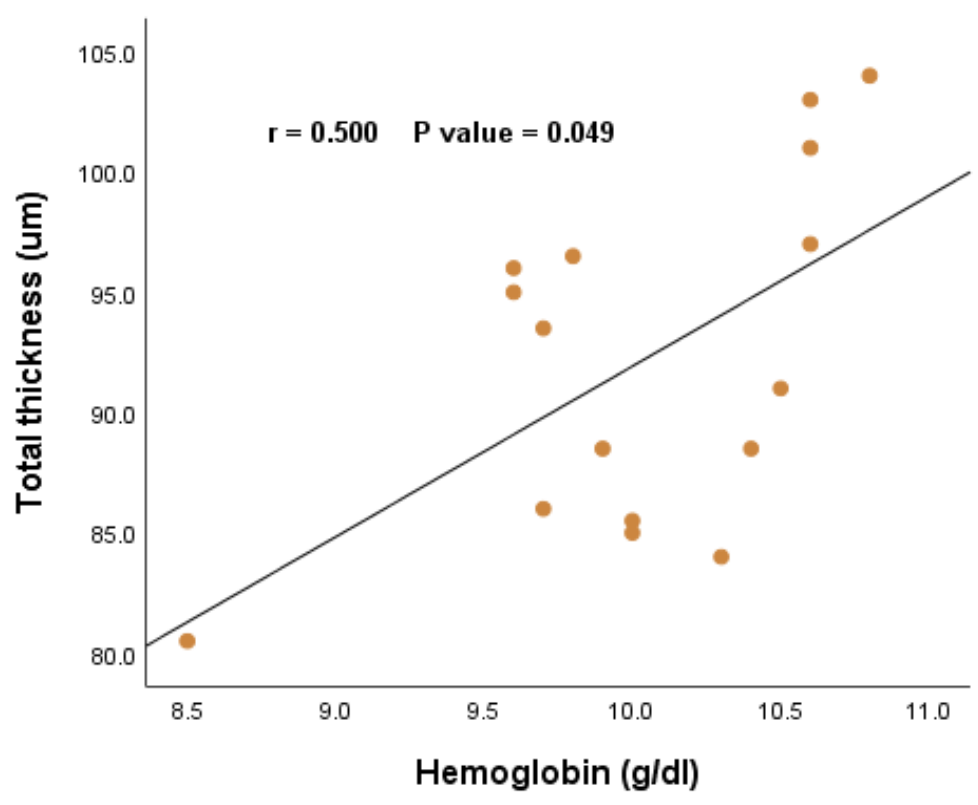

Figure (2) Correlation between total thickness and hemoglobin

There were no significant correlations between peripapillary RNFL thicknesses and the other hematologic parameters (serum iron, ferritin, TIBC and MCV) in the anemic group, except that mentioned before.

\section{Discussion}

A previous study reported that women with iron deficiency anemia had significantly smaller RNFL thicknesses in the nasal and inferior quadrants compared with healthy matched subjects (6). Another study was conducted on 40 children with a diagnosis of
IDA, and it was found that average RNFL and RNFLs of superior and inferior quadrants were significantly thinner in the anemic children than in the healthy children (4).

The present study demonstrated total average RNFL and RNFL of inferior, superior, nasal and temporal quadrants were thinner in children with IDA than in healthy children ( $\mathrm{P}$ value <0.001, <0.001, 0.001, 0.002 and 0.011 respectively), according to measurements performed by Topcon 3D OCT-2000 $\mathrm{Fa}$, and these results are 
consistent with the findings of recent OCT studies (4) (6) (8), moreover we could detect that the temporal RNFL thickness was also thinner in anemic children. In addition, average peripapillary RNFL thickness and RNFL thicknesses of inferior, superior, and temporal quadrants were correlated with hemoglobin levels in anemic children $(\mathrm{r} 1=$ $0.500, \mathrm{P} 1<0.049, \mathrm{r} 2=0.560, \mathrm{P} 2<0.024, \mathrm{r} 3$ $=0.506, \mathrm{P} 3<0.046, \mathrm{r} 4=0.654, \mathrm{P} 4=0.006$, respectively).

Contrary to the findings of two previous studies that showed significant correlation between peripapillary RNFL thickness and hematological parameters; serum iron, ferritin concentration and total iron binding capacity (6) (8), another studies showed no correlation between RNFL thickness and other hematological parameters $(4)(7)$.

Speaking of that, the present study showed a significant correlation between MCV and total RNFL thickness and inferior quadrant $(\mathrm{r} 1=0.554, \mathrm{P} 1=0.026$ and $\mathrm{r} 2=$ $0.578, \mathrm{P} 2=0.019$ respectively), and the iron level was correlated, in anemic children, with nasal quadrant only $(r=0.528 \& \mathrm{P}$ value<0.036).

Indeed, one published study attributed the study findings to dopaminergic dysfunction as known dopamine is a major neurotransmitter in the retina, and its deficiency is believed to causes modification in receptive field properties of ganglion cells which form RNFL by their axons. Also, they noticed that the pattern of RNFL thinning in children with IDA could mimic the pattern found in glaucoma due to the superior and inferior quadrant predilection (4).

Speaking of the study results mentioned above, as it was conducted on children with IDA, the current study obtained; a lower average of RNFL in anemic children than the mentioned above (4). (92.2 um and 98.6 um respectively) and slightly higher average of RNFL in control group (107.8 um), compared with the control group (102.0 um) of published study. Also, in this study compared to the previous study (4); the inferior quadrant was thinner in anemic group (110.9 um) compared with (123.7 um), the superior quadrant was thinner in anemic group (112.4 um) compared with (123.1 um), the nasal quadrant was slightly thinner in anemic group (75.4 um) compared with (79.4 um) and the temporal quadrant was slightly thicker (70.2 um) compared with (68.3 um) respectively. Regarding the control group; the inferior quadrant was thicker (137.4 um) compared with (132.5 um), the superior quadrant was slightly thinner (127.8 um) 
compared with (129.8 um), the nasal quadrant was thicker (86.7 um) compared with (77.0 um) and the temporal quadrant was thicker (80.2 um) compared with (68.9 um).

Iron is needed as cofactor for cytochromes and the oxidative chain and hence for ATP production, also needed for many of the enzymes that responsible for cholesterol and fatty acid synthesis, which are precursors of myelin. These enzymes are enriched in oligodendrocytes compared to other cells, the net result of iron deficiency is impairment of energy production and hence decrease in myelin production (9).

A previous meta-analysis study showed that the RNFL thickness, in obstructive sleep apnea syndrome (OSAS) patients, is much thinner than healthy population, especially in superior and inferior quadrant. In patients with OSAS, intermittent upper airway obstruction occurs and result in hypoxia and decrease in $\mathrm{PaO} 2$ (10).

In the current, study the strong correlation between the hemoglobin level and RNFL thickness suggest that the hypoxic effect of anemia is more influenced in RNFL thinning more than iron deficiency element (iron level was correlated with nasal quadrant only), however the effect of iron deficiency on oligodendrocytes myelination and their function couldn't be ignored and may exaggerate the insult of anemic hypoxia.

\section{Conclusion}

Our study has shown the average RNFL and RNFL of inferior, superior, nasal and temporal quadrants were thinner in children with IDA than in healthy children, in addition, average peripapillary RNFL thickness and RNFL thicknesses of inferior, superior, and temporal quadrants were correlated with hemoglobin levels in anemic children. That measures were obtained with Topcon 3D spectral domain OCT $2000 \mathrm{Fa}$.

Therefore, we caution physicians especially ophthalmologists to the impact of IDA on the eye, as it is associated with RNFL thinning. And so, a special attention should be considered while obtaining a normative data in children age group and hence the diagnosis of other ophthalmic diseases that rely on RNFL measurements such as glaucoma. Also, we recommend a further study to demonstrate the effect of iron replacement therapy and treatment of IDA on RNFL.

\section{References}

1. Cusick, S., Georgieff, M., \& Rao, R. (2018). Approaches for reducing the risk of early-life 
iron deficiency-induced brain dysfunction in children. Nutrients, 10(2), 227.

2. Simsek, A., Tekin, M., Bilen, A., Karadag, A. S., Bucak, I. H., \& Turgut, M. (2016). Evaluation of Choroidal Thickness in Children With Iron Deficiency Anemia. Investigative ophthalmology $\&$ visual science, 57(14), 5940-5944 .

3. Lozoff, B. (2011). Early iron deficiency has brain and behavior effects consistent with dopaminergic dysfunction. The Journal of nutrition, 141(4), 740S-746S.

4. Türkyilmaz, K., Öner, V., Özkasap, S., Şekeryapan, B., Dereci, S., \& Durmuş, M. (2013). Peripapillary retinal nerve fiber layer thickness in children with iron deficiency anemia. European journal of ophthalmology, 23(2), 217-222.

5. Cumurcu, T., Firat, P. G., Çavdar, M., Doğanay, S., \& Kuku, İ. (2011). Macular Hemorrhage as a Complication of Iron Deficiency Anemia. Journal of Retina-Vitreous, 19(4), 093-095.

6. Akdogan, E., Turkyilmaz, K., Ayaz, T., \& Tufekci, D. (2015). Peripapillary retinal nerve fibre layer thickness in women with iron deficiency anaemia. Journal of International Medical Research, 43(1), 104-109.

7. Oncel Acir, N., Dadaci, Z., Cetiner, F., Yildiz, M., Alptekin, H., \& Borazan, M. (2016). Evaluation of the peripapillary retinal nerve fiber layer and ganglion cell-inner plexiform layer measurements in patients with iron deficiency anemia with optical coherence tomography. Cutaneous and ocular toxicology, 35(2), 131136.

8. Cikmazkara, I., \& Ugurlu, S. K. (2016). Peripapillary retinal nerve fiber layer thickness in patients with iron deficiency anemia. Indian journal of ophthalmology, 64(3), 201.

9. Todorich, B., Pasquini, J. M., Garcia, C. I., Paez, P. M., \& Connor, J. R. (2009). Oligodendrocytes and myelination: the role of iron. Glia, 57(5), 467-478.

10. Sun, C. L., Zhou, L. X., Dang, Y., Huo, Y. P., Shi, L., \& Chang, Y. J. (2016). Decreased retinal nerve fiber layer thickness in patients with obstructive sleep apnea syndrome: A metaanalysis. Medicine, 95.(rr).

To cite this article: Abdelmonem M. Hamed, Mohamed R. El Sayed, Mohamed A. Heikal, Ehsan Abd Al Basset. Evaluation of Peripapillary Retinal Nerve Fiber Layer by Optical Coherence Tomography in Children with Iron Deficiency Anemia. BMFJ 2022;39 (ophthalmology):108:116. DOI: 10.21608/bmfj.2021.18447.1112 